\title{
EDEMA PULMONAR DE REEXPANSÃO TRATADO COM VENTILAÇÃO NÃO INVASIVA: RELATO DE CASO
}

\author{
REEXPANSION PULMONARY EDEMA TREATED WITH NON-INVASIVE \\ VENTILATION: CASE REPORT
}

\author{
Amarilio Vieira de Macedo Neto, ACBC-RS ${ }^{1}$ \\ Gémerson Gabiatti ${ }^{2}$ \\ Jair Garcia da Silva ${ }^{2}$
}

\section{INTRODUÇÃO}

O edema pulmonar de reexpansão (EPR) constitui complicação potencialmente severa pós-drenagem pleural em determinadas situações. $\mathrm{O}$ reconhecimento precoce de suas manifestações e das situações que freqüentemente o desencadeiam é fundamental para reduzir a morbi-mortalidade associada.

\section{RELATO DO CASO}

Paciente masculino, branco, 34 anos foi atendido no Hospital de Pronto-socorro Municipal de Porto Alegre (HPS) queixando-se de dispnéia leve, dor torácica em hemitórax direito e tosse produtiva há uma semana. Negava episódio semelhante no passado e tabagismo. Ao exame, apresentava bom estado geral, encontrava-se eupnéico, acianótico e com sinais vitais normais. A ausculta pulmonar evidenciava importante diminuição do murmúrio vesicular em todo hemitórax direito. Uma radiografia de tórax demonstrava grande câmara de pneumotórax à direita (Figura 1).

$\mathrm{O}$ paciente foi submetido à drenagem pleural direita através de um dreno $36 \mathrm{~F}$ conectado a um sistema em selo d'água. $\mathrm{O}$ procedimento transcorreu sem complicações. $\mathrm{O}$ estudo radiológico pós-drenagem evidenciava reexpansão pulmonar completa. Três horas após iniciou com agitação, dispnéia intensa, tosse e expectoração de secreção rósea. Recebeu oxigênio suplementar por cateter nasal a 31/min, mas mesmo assim a oximetria de pulso identificava a saturação da hemoglobina entre $85 \%$ e $88 \%$. Neste momento auscultavam-se estertores crepitantes difusos no pulmão direito e uma nova radiografia mostrava reexpansão pulmonar completa e infiltrado pulmonar difuso com importante diminuição da transparência homolateral (Figura 2).

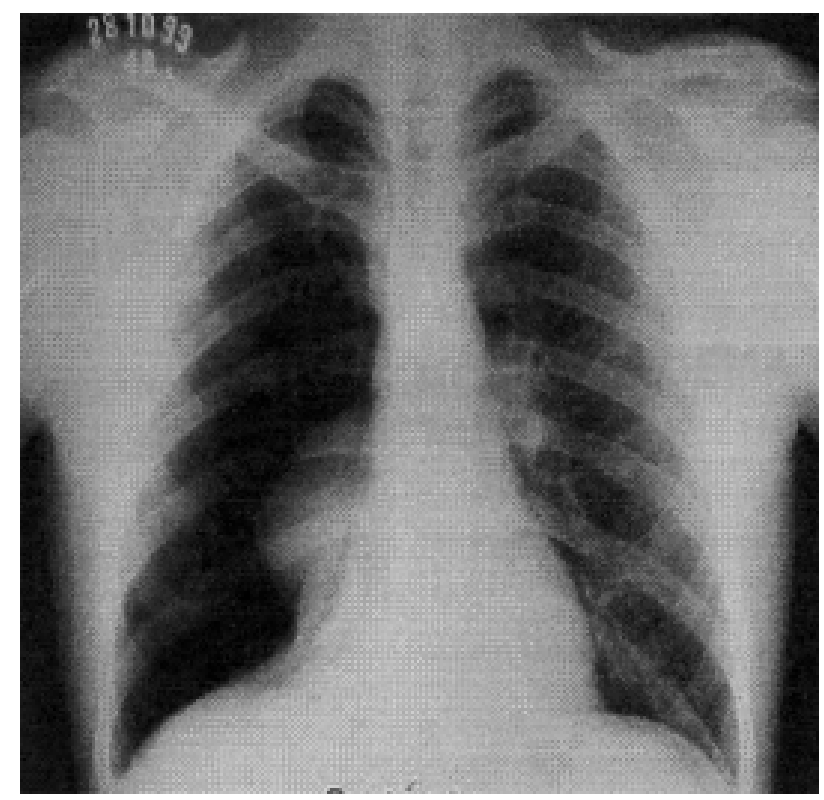

Figura 1 - Grande pneumotórax à direita.

A gasometria arterial neste momento demonstrava $\mathrm{pH}$ de 7,35 , pressão parcial arterial de oxigênio $\left(\mathrm{paO}_{2}\right)$ de $44 \mathrm{mmHg}$, saturação arterial da hemoglobina $\left(\mathrm{SaO}_{2}\right)$ de $77 \%$ e pressão parcial arterial de dióxido de carbono $\left(\mathrm{paCO}_{2}\right)$ de $36 \mathrm{mmHg}$. Os sinais vitais eram os seguintes: freqüência cardíaca (FC) de 130 batimentos por minuto (bpm), pressão arterial de 100/80 milímetros de mercúrio (mmHg) , temperatura axilar de 37.6 graus Celsius $\left({ }^{\circ} \mathrm{C}\right)$ e freqüência respiratória (FR) de 40 movimentos por minuto (mpm). A drenagem até o momento havia sido de $100 \mathrm{ml}$ com aspecto sero-hemático e o hemograma demonstrava uma he-

1. Professor Adjunto da Faculdade de Medicina da UFRGS. Preceptor responsável pela Equipe de Cirurgia Torácica Programa de Residência em Cirurgia do Trauma do HPS-POA. Doutor em Medicina pela Universidade Federal do Rio de Janeiro.

2. Cirurgião Geral. Residente em Cirurgia do Trauma do HPS-POA.

Recebido em 22/3/2000

Aceito para publicação em 17/7/2000

Trabalho Realizado no Hospital de Pronto-socorro de Porto Alegre (HPS-POA) - Residência em Cirurgia do Trauma. 
moglobina $(\mathrm{Hb})$ de 20,6g/dl e leucócitos totais de 28.600 com $4 \%$ de bastões. Com a hipótese de edema pulmonar pós-reexpansão foi encaminhado para a UTI, onde foram instituídas as seguintes medidas: ventilação não invasiva por pressão positiva contínua na via aérea (CPAP) através de máscara facial com oclusão nasal e oral, conectada a ventilador. Foram administrados conjuntamente $5 \mathrm{mg}$ de morfina, $80 \mathrm{mg}$ de furosemida e $240 \mathrm{mg}$ de aminofilina intravenosa. Para analgesia foi aplicado via intramuscular $75 \mathrm{mg}$ de diclofenaco.

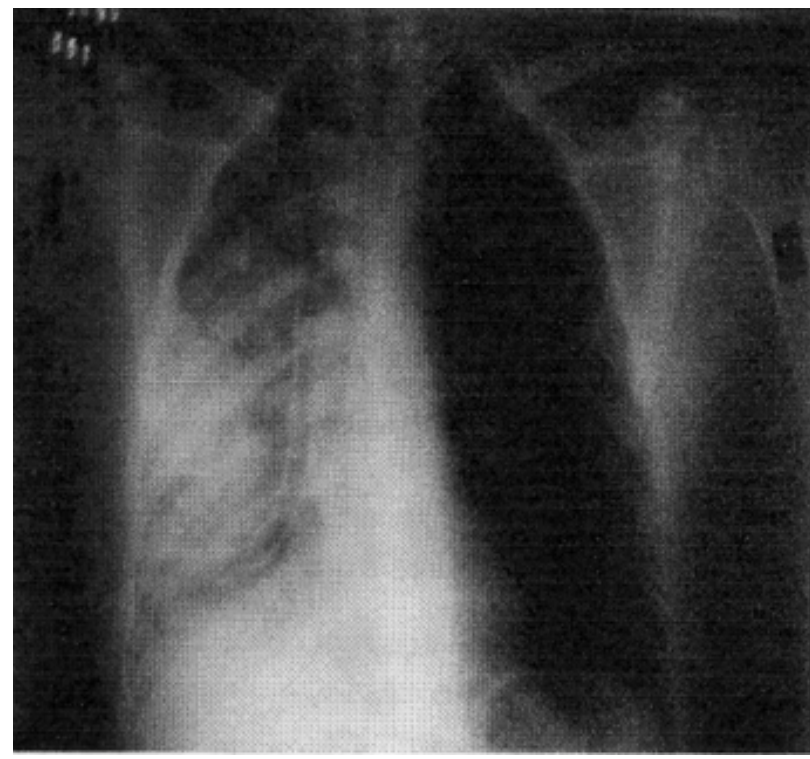

Figura 2 - Infiltrado pulmonar importante no lado drenado.

O quadro clínico manteve-se estável com boa tolerância do paciente à ventilação não invasiva, que permaneceu por 12 horas. Os sinais eram: pressão arterial 100/ $80 \mathrm{mmHg}$ com variações da FC de 90 a $110 \mathrm{bpm}$ e da FR de 20 a $24 \mathrm{mpm}$, embora mantivesse hipoxemia ( gasometria $=\mathrm{pH}: 7,34, \mathrm{paO}_{2}: 50,3 \mathrm{mmHg}, \mathrm{SaO}_{2}: 82 \%, \mathrm{paCO}_{2}:$ 40mmHg). O hemograma mostrava: Hb: 21g/dl, Leucócitos: 34.200 com $1 \%$ de bastões. O quadro radiológico mantinha-se inalterado. $\mathrm{O}$ paciente evoluiu favoravelmente, tendo sido transferido para a enfermaria em 48 horas depois e teve alta hospitalar no 3 - dia. Retornou para revisão ambulatorial duas semanas após, sem queixas. O radiograma de tórax de controle mostrava apenas pequenas áreas atelectásicas residuais.

\section{DISCUSSÃO}

O EPR constitui complicação rara da reexpansão de um pulmão colapsado ${ }^{1}$. Tipicamente, ocorre após a drenagem de um grande derrame pleural ou pneumotórax, especialmente se o quadro estiver estabelecido por um longo período (mais de três dias) ${ }^{2,3}$, embora possa ocorrer com menos tempo. Na maioria dos casos o EPR distribui-se difusamente sobre a área reexpandida, sendo rara a apresen- tação localizada. O EPR foi descrito inicialmente no século XIX como expectoração albuminosa após drenagem de derrame pleural. Em 1958, descreveu-se sua ocorrência após a evacuação de um pneumotórax. Sua incidência em algumas séries varia de zero a $27 \%{ }^{1}$ podendo determinar taxas de mortalidade ao redor de $20 \%{ }^{4}$. Portanto, é uma grave intercorrência, e deve ser tratada prontamente.

As manifestações clínicas do EPR variam desde a apresentação assintomática com anormalidades radiográficas isoladas até abrupta e grave insuficiência respiratória e comprometimento hemodinâmico. Em dois terços dos casos clinicamente manifesta-se por: dispnéia, taquipnéia, taquicardia, cianose, tosse seca ou com eliminação de secreção rosácea, hemoptise franca, dor torácica, hipotensão e sudorese iniciando até uma hora após a expansão pulmonar. Usualmente o quadro é autolimitado evoluindo com melhora espontânea em alguns dias. Ocasionalmente, a hipoxemia é intensa, podendo progredir para insuficiência respiratória grave, choque e parada cardíaca ${ }^{4}$.

A etiologia do EPR é controversa. Os mecanismos propostos incluem:

1. Aumento da pressão hidrostática devido à inundação vascular do pulmão reexpandido conseqüente à pressão negativa intrapleural;

2. Alteração da permeabilidade vascular devido ao estiramento da microcirculação pulmonar;

3. Alteração da tensão superficial alveolar secundário à diminuição de surfactante;

4. Alteração da permeabilidade da membrana vascular pulmonar devido à hipóxia no segmento atelectasiado.

Algumas evidências clínicas apontam para o último mecanismo como mais provável ${ }^{1,2,3,4}$. Índices de proteínas no escarro comparados aos níveis séricos variam de 0,74 a 0,85 , favorecendo a teoria do aumento da permeabilidade capilar em relação ao edema hidrostático, cuja taxa de proteína edema/soro seria menor, em torno de $0,6^{4}$. Acreditase que durante o período colapsado a hipóxia tecidual resulte em diminuição do oxigênio mitocondrial e aumento do metabolismo anaeróbico, ocasionando aumento nos níveis intracelulares de duas enzimas: xantina oxidase e aldeído oxidase. Estas enzimas, após a reexpansão e reintrodução do oxigênio nas zonas hipóxicas, produziriam um aumento na produção de radicais livres, os quais danificariam o epitélio alveolar endotelial, produzindo aumento da permeabilidade vascular ${ }^{1,3,4}$.

Nos quadros leves, o oxigênio suplementar, aliado à restrição hídrica e salina e ao uso de diuréticos são as medidas terapêuticas necessárias no tratamento do EPR. Entretanto, nas situações mais graves, com hipoxemia acentuada, o objetivo do tratamento é o de reduzir o shunt intrapulmonar. $\mathrm{O}$ tratamento clássico envolve ventilação mecânica invasiva e pressão expiratória final positiva uma vez que esta limita o aumento de líquido pulmonar extravascular e aumenta o fluxo linfático através do ducto torácico. A ventilação mecânica não invasiva com pressão positiva aplicada através de máscaras, nasal ou facial é uma alternativa elegante e menos agressiva. Tem sido usada desde o inicio da década de 1990 para o tratamento 
de pacientes com edema pulmonar cardiogênico, tanto no tratamento da fase aguda da insuficiência respiratória como em domicílio em pacientes com insuficiência cardíaca congestiva. A ventilação por máscara é empregada através de um sistema de fluxo contínuo ou intermitente oferecido pelo próprio ventilador mecânico ou gerador de fluxo próprio para uso domiciliar. Usualmente a pressão positiva na via aérea é administrada de forma contínua (continuous positive airway pressure - CPAP) ou na ins e expiração (BIPAP). No caso apresentado, foi utilizada ventilação com pressão positiva contínua (CPAP) através da máscara facial conectada a ventilador.
Ventilação não invasiva traz vantagens quando comparada com a forma invasiva desde que o paciente tenha boa

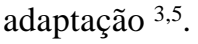

A utilização de válvulas no sistema de drenagem torácica para controlar o fluxo, promovendo uma reexpansão pulmonar mais lenta é descrita como opção para reduzir a incidência do EPR. Entretanto não há evidências claras que sustentem tal proposição e inúmeros casos de EPR são descritos mesmo com a utilização de tal técnica ${ }^{1,4}$. A reavaliação clínica periódica nas primeiras horas pós-drenagem torácica de grandes coleções com mais de 72 horas de evolução deve identificar aqueles com maior risco.

\begin{abstract}
The authors report a case of Reexpansion Pulmonary Edema (RPE) seen at Hospital de Pronto Socorro de Porto Alegre 3 hours after drainage of spontaneous pneumothorax. The patient presented a unilateral pneumothorax with one-week duration. After pleural drainage respiratory failure occured being managed at the Intensive Care Unit with non-invasive positive pressure ventilation through facial mask. The patient had favorable outcome and was discharged asymtomatic after 72 hours.
\end{abstract}

Key Words: Reexpansion pulmonary edema; Pleural drainage; Non-invasive ventilation.

\title{
REFERÊNCIAS
}

1. Matsuura Y, Nomimura T, Murakami H et al - Clinical analysis of reexpansion pulmonary edema. Chest, 1991; 100(6), 1562-6.

2. Waqarunddin M, Bernstein A - Reexpansion pulmonary oedema. Thorax, 1975; 30(1), 54-60.

3. Aide MA, Judice LF, Cordeiro PB et al. Edema pulmonar de reexpansão. Arq Bras Med, 1990; 64(4), 245-7.

4. Woodring JH - Focal reexpansion pulmonary edema after drainage of large pleural effusions: clinical evidence suggesting hypoxic injury to the lung as the cause of edema. South Med J, 1997; 90(12), 1176-82.
5. Antonelli M, Conti G, Rocco M et al. A comparison of noninvasive positive-pressure ventilation and conventional mechanical ventilation in patients with acute respiratory failure. N England J Med, 1998; 339, 429-35.

Endereço para correspondência:

Dr. Amarílio Vieira de Macedo Neto

Hospital de Pronto-socorro de Porto Alegre

Assessoria Científica - Res. Cirurgia do Trauma

Largo Teodoro Hertz, S/N - 1ํandar

90040-192 - Porto Alegre-RS

E-mail: rcthps@hotmail.com 